\title{
Stochastic Screening Dithering with Adaptive Clustering
}

\author{
Luiz Velho \\ Jonas Gomes \\ IMPA - Instituto de Matemática Pura e Aplicada ${ }^{1}$
}

\begin{abstract}
We develop a clustered dithering method that uses stochastic screening and is able to perform an adaptive variation of the cluster size. This makes it possible to achieve optimal rendition of gray shades while preserving image details. The algorithm is an improvement to the dithering with space filling curves method, published in [3].
\end{abstract}

CR Descriptors: B.4.2 [Input/Output and Data Communications]: Input-Output Devices - Image Display; I.3.3 [Computer Graphics]: Picture/image generation - Display algorithms; I.3.6 [Computer Graphics]: Methodology and Techniques; I.4.3 [Image Processing]: Enhancement.

Additional Keywords: Digital Halftoning, Dithering Algorithms, Space Filling Curves, Adaptive Clustering.

\section{INTRODUCTION}

The reproduction of gray scale images in bilevel graphic display devices is achieved through a process called halftoning. Given a gray scale image it consists in generating a binary image which perceptually, approximates the original image. This process can be analog or digital. Digital halftoning is done using a technique called dithering to determine the binary state (black or white) of the elements of the output image. Dithering algorithms distribute the black and white pixels in such a way that the input and output images are perceptually as close as possible, within the physical limitations of the display device.

Dithering methods can be subdivided into two main groups according to the type of images they produce [2]. Dispersed dot dithering methods generate images in which black and white pixels are evenly distributed throughout the image area. Clustered dot dithering methods generate images in which black and white pixels are concentrated together forming clusters. These two approaches serve different purposes and are suitable for different classes of display devices.

Classical clustering dithering algorithms use regular, fixed size cluster, and the black and white dot patterns inside the cluster vary

\footnotetext{
${ }^{1}$ IMPA - Instituto de Matemática Pura e Aplicada, Estrada Dona Castorina, 110 22460-320 Rio de Janeiro, RJ, Brazil, Ivelho I jonas@visgraf.impa.br
}

Permission to make digital/hard copy of part or all of this work for personal or classroom use is granted without fee provided that copies are not made or distributed for profit or commercial advantage, the copyright notice, the title of the publication and its date appear, and notice is given that copying is by permission of ACM, Inc. To copy otherwise, to republish, to post on servers, or to redistribute to lists, requires prior specific permission and/or a fee.

(C) 1995 ACM-0-89791-701-4/95/008 ...\$3.50 according to the image intensity values. These clusters are distributed periodically over the image domain. For this reason, these algorithms are known by the name of amplitude modulated (AM) dithering.

Stochastic dithering algorithms use a fixed size dot pattern and vary their spacing according to the image intensity values. For this reason, these algorithms are called frequency modulated (FM) dithering. Frequency modulated dithering techniques have been implemented recently on the raster image processors of high resolution phototypesetters.

The space filling curve dithering algorithm introduced in [3], contains characteristics of both AM and FM dithering: it is a clustered dithering technique, but the clusters are distributed stochastically over the image domain. Therefore the algorithm adapts well to a wide range of display devices.

Figure 1 describes the space of possibilities covered by the existing digital halftoning techniques: from periodic clustered dither, to periodic dispersed dot dither, and from this to aperiodic dispersed dot dithering techniques.

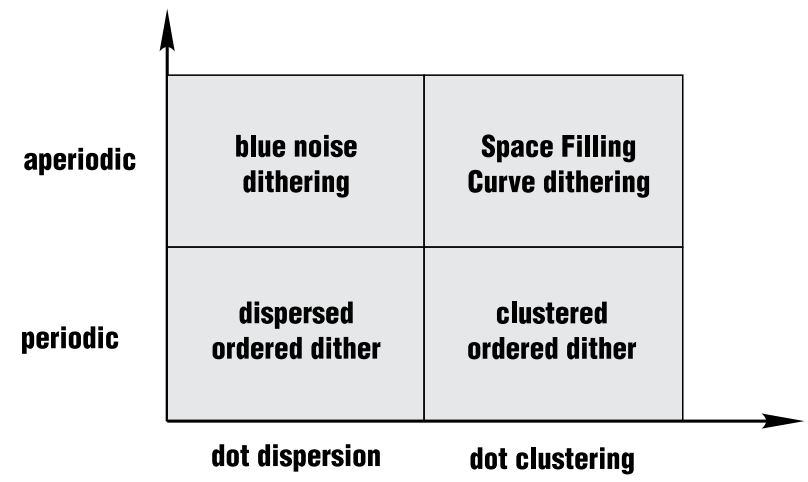

Figure 1: Range of existing dithering techniques.

In this paper we present an improvement to the cluster construction of the algorithm in [3] in order to get a better rendition of image details, without loosing tonal resolution. The major result though, is an extension of the algorithm to obtain a dispersed clustered dithering technique whose cluster size may vary adaptively according to image intensity characteristics. We should point out that this extension was suggested in [3].

An earlier report of the method presented in this paper containing some preliminary results and simple experiments appeared in [4]. Here, we formulate the problem from the point of view of 
AM/FM dithering techniques and investigate the application of the algorithm to image reproduction on high resolution bilevel devices.

The remaining of the paper is organized as follows: Section 2 reviews the space filling curve dither algorithm. Section 3 describes the dither with adaptive clustering. Section 3.1 discusses implementation related issues. Section 4 shows results of experiments with the method.

\section{SPACE FILLING CURVE DITHER}

In this section we briefly review the dithering with space filling curves (SFC) introduced in [3]. The trace of a space filling curve approximation is used to scan the image, generating a parametrization of the image elements with many desirable properties.

The method consists of the following steps:

- Subdivision of the image into cells;

- Computation of the average intensities of each cell;

- Generation of corresponding black and white dot patterns for each cell;

- positioning of the dot pattern within the cell to generate the cluster.

The subdivision of the image is performed by following the path of the space filling curve until the number of elements visited is equal to the cluster size. This is illustrated in Figure 2(a), using a Hilbert space filling curve.

For each cell, the computation of the accumulated intensity is performed as each one of its elements is visited. Then, the corresponding dot pattern is generated by selecting a group of contiguous elements proportional in number to the total intensity. In this way, the cell is subdivided into subcells of black and white pixels such that its average intensity approximates the image average intensity within the cell. Figure 2(b), from [3], shows the configuration of dots corresponding to intensity levels $15 / 16$ to 0 for a cluster of $4 \times 4$ pixels.

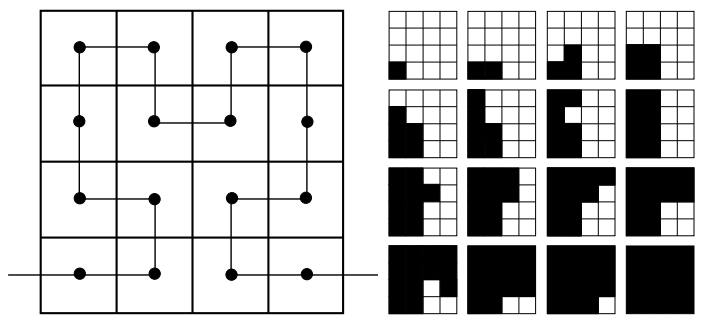

(a)

(b)

Figure 2: $4 \times 4$ cluster and dot patterns - from (Velho and Gomes, 1991).

There is a quantization error associated with each cell defined by the difference between the average intensity of the grayscale image and the intensity of the dot patterns. The quantization error in a region is diffused by the algorithm, propagating it to neighbor regions along the path of the space filling curve.

\subsection{Cluster Generation}

The last step of the algorithm creates the cluster by positioning the dot pattern within the cell. Here we introduce an improvement over the algorithm published in [3]. We position the central pixel of the generated dot pattern at the pixel inside the cell which has the lowest intensity level (i.e. corresponding to the highest percentage of black ink). This is illustrated in Figure 3: (a) shows the image and a $4 \times 4$ cell; (b) shows (in gray) the lowest intensity pixel within the cell; (c) shows a dot pattern of 5 pixels; (d) shows the translation of the dot pattern center to the pixel of lowest intensity. This positioning method results in a much better rendition of the image details, without compromising tonal resolution.

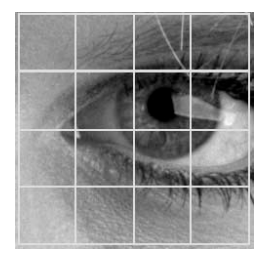

(a)

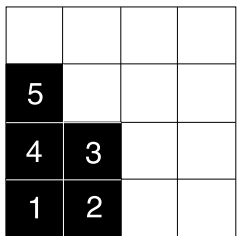

(c)

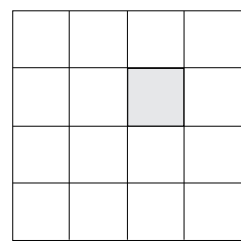

(b)

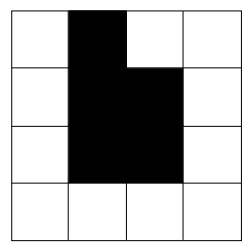

(d)
Figure 3: Dot pattern position.

We should observe that besides the non-directionality implied by the space filling curve traversal of the image pixels, the cluster generation method described introduces some randomness to the distribution of the dot patterns within the cell.

In brief, the dithering algorithm with space filling curves uses clustering similar to the traditional amplitude modulated (AM) algorithms, but at the same time it performs error diffusion. Therefore, it also incorporates characteristics of techniques that use frequency modulation (FM dithering).

\section{SFC DITHER with ADAPTIVE CLUSTERING}

From the results of the previous section we know that the dithering algorithm with space filling curves distributes stochastically the clusters and performs an error diffusion between neighbor cells. In this section we will show how to extend the method in order to have an adaptive control over the cluster size. This control will enable us to incorporate a variable size clustering, which along with the above mentioned properties, creates a dithering texture similar to the film grain found in photographic textures.

The space filling curve dithering algorithm subdivides the image domain into cells, and at each cell it approximates the image function $f(x, y)$ by some bi-level image function $\bar{f}(x, y)$. The approximation criteria is a perceptual one, based on pixel intensities. The adaptive clustering dithering consists of changing the size of each cell, based on some adaptive criteria, in order to get a better binary approximation $\bar{f}$ of the image function $f$.

The adaptiveness criteria to compute the cluster size depends on the desired effect to be obtained by the halftone method. In our case, the goal is to achieve the best rendition of image detail without compromising tonal reproduction. Therefore, we should use an adaptive criteria that varies the cluster size according to the rate of change of the image intensity. In order to accomplish for this, we need to measure the variation of image intensities as we scan the image. 
Since we are scanning the image along the path of the space filling curve, the directional derivative along the curve provides a good measure ot the rate of change of the image intensities along the scanning direction. It is computed by modulating the gradient vector

$$
\operatorname{grad} f=\left(\frac{\partial f}{\partial x}, \frac{\partial f}{\partial y}\right)
$$

of the image function $f$, by the unit vector $u$ along the scanning direction defined by the space filling curve. That is,

$$
\frac{\partial f}{\partial u}=\langle u, \operatorname{grad} f\rangle
$$

where $\langle$,$\rangle is the usual euclidean inner product.$

After deciding that the directional derivative will take care of the adaptiveness criteria, it remains to obtain the correct relationship between the cluster size and the derivative intensity. As the derivative magnitude gets bigger, image intensities change faster and, therefore, the cluster size should get smaller.

We need to find the correct relationship between the cluster size and the derivative values. For this, we first observe that the intensities distribution in a dithered image must follow a perceptual criteria. Also, the eye response to intensity changes obeys a logarithmic law (see [1]). Based on these two remarks, we conclude that we should vary the cluster size exponentially with the gradient magnitude. This rule maintains a linear relationship between the perceptual intensity inside each cluster and the directional slope of the image intensity.

\subsection{Implementation Issues}

It is possible to implement the space filling curve dithering with adaptive cluster in two stages:

\section{Estimate the cluster size based on image characteristics;}

\section{Change the cluster size according to some function of (1).}

The separation of these two mechanisms makes the algorithm more flexible and allows for experimentation with different sets of criteria in the specification of the cluster size. The latter procedure gives input to the former establishing a clean interface between them.

Although in this work we have experimented mainly with an adaptive criterium based on the variation of the directional derivative of the image intensity function, as explained in the previous section; there are other types of criteria that we believe are worth exploring. One example is the cluster adaptation based on the physical reproduction function of the imaging system in order to compensate for its deficiencies. Other example is an adaptation criterium based on a function of the image domain in order to create graphical effects. This opens up many other possibilities of use for the method.

In our implementation the cluster size control is done in the first pass through the cluster cells. At each point of the image, the maximum allowable cluster size is determined by the adaptation criteria. While image elements in the region are processed (to accumulate intensity), the current cluster size is compared with the maximum allowable cluster size at that point. If this maximum allowable size is smaller than the current size, it becomes the current cluster size. The algorithm terminates the first pass when the number of elements in the region exceeds the cluster size. Then, it moves on to generate the dot pattern configuration, as explained in the previous section.

To make the algorithm more versatile, the adaptation criteria can be passed as a parameter to the program. The default criterium is the rule described above using an exponential of base 2 . The other options provided include: linear variation with the value of the directional derivative of the image function, or using a table supplied by the user.

\section{RESULTS}

In this section we show the results of applying the algorithm to various types of images. These test images reflect the main characteristics of images encountered in digital printing situations.

The figures below compare the output of the space filling curve dithering algorithm with and without adaptive clustering. All input images are gray-scale with 8 bits of intensity resolution and spatial resolution varying from 80 to $400 \mathrm{ppi}$ (pixels per inch). The output images are halftoned and printed at $1200 \mathrm{dpi}$ (dots per inch). The images in Figures 4 and 5 are scaled using pixel replication in order to show more clearly the halftone screen dots.

Figure 4 is a test pattern of intensity gradations using linear ramps. The gradient increases in steps such that the rate of change in intensity is almost constant for each rectangle. As the slope of the ramp doubles, the cluster size also decreases by a factor of two. The maximum cluster size is 31 pixels.

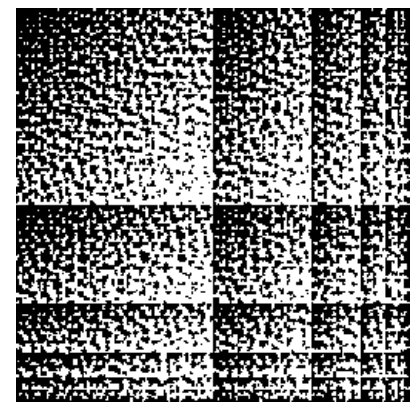

(a)

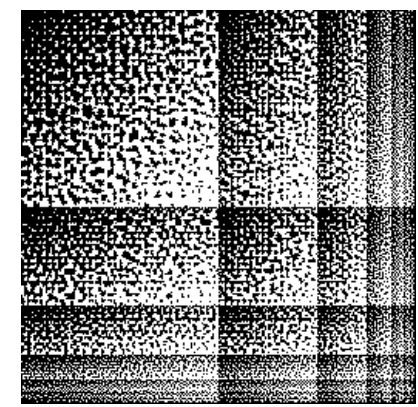

(b)
Figure 4: Linear intensity gradations (a) constant cluster size of 31 pixels (b) variable cluster size.

Figure 5 is a cartoon image consisting of line drawings and areas of constant gray level. The shadow on the wall in the background is rendered as regular pattern of dots simulating a standard halftone screen. The effect of the adaptive method is striking. The improvement obtained is mainly due to the high frequencies of the image. It needs dithering only in the areas of intermediate intensity. In this case, the algorithm was capable of matching exactly the edges of the drawings and at the same time reproducing with uniform dot patterns the different gray shades. We should remark that printing this image with the traditional AM clustering algorithm would certainly result in moirée patterns on the gray regions of the original image, because of the dot patterns used to produce the gray shade.

Figure 6 shows a comparison of the adaptive dithering (right) with the space filling curve dithering algorithm using fixed cluster size (left), as we increase the cluster size. The fixed cluster sizes in this figure are, from top to bottom, 30, 15 and 3 pixels. Also, these are the maximum cluster sizes used to process the corresponding image with the adaptive algorithm.

Finally, we show the application of the algorithm for high resolution printing. In order to do this, we made several tests to find the optimal cluster size for the phototypesetter used to print this paper. The image in Figure 7(b) is printed at 1200 dpi with a maximum cluster size of 7 pixels, as determined by the experiments. The image in Figure 7(a) is printed at the same resolution with a dispersed-dot dither (cluster size of 1 pixel). This comparison demonstrates the effectiveness of the method to match the characteristics of the output device.

\section{CONCLUSIONS}

An adaptive digital halftoning method with variable size clustering was presented. It extends the space filling curve dithering algorithm 


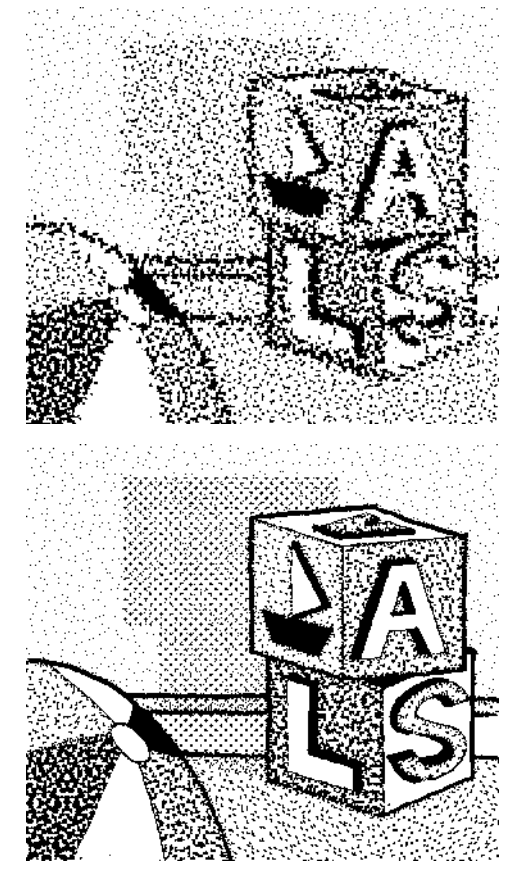

Figure 5: Line drawing cartoon (a) constant cluster size of 27 pixels (b) variable cluster size

to change the size of pixel clusters according to local characteristics of the image. This makes possible to achieve an optimal rendition of gray shades while preserving image detail.

The dithering techniques described in this paper can also be applied with great effectiveness to color images. We developed a color halftoning algorithm that employs adaptive dot clustering with error diffusion on multiple image channels [5]. These features make the algorithm particularly suited for color reproduction using any number of process colors.

\section{ACKNOWLEDGEMENTS}

This work was done during the period the authors visited the Hewlett-Packard Labs in Palo Alto. We wish to thank Ricardo Motta who provided us with the working environment necessary for doing this research. The visit to HP was sponsored by the RHAE project from MCT/CNPq in Brazil.

\section{REFERENCES}

[1] A. Rosenfeld and A. C. Kak. Digital Picture Processing. Academic Press, 1976.

[2] R. Ulichney. Digital Halftoning. MIT Press, Cambridge, Ma, 1987.

[3] L. Velho and J. de M. Gomes. Digital halftoning with space filling curves. Computer Graphics (Proceedings SIGGRAPH '91), 25(4):81-90, 1991.

[4] L. Velho and J. de M. Gomes. Space filling curve dithering with adaptive clustering. In Proceedings of SIBGRAPI'92, V Brazilian Simposium of Computer Graphics and Image Processing, pages 1-9. SBC - Sociedade Brasileira de Computação, 1992.

[5] L. Velho and J. de M. Gomes. Color halftoning with stochastic screening and adaptive clustering. Preprint, 1995. IMPA Instituto de Matemática Pura e Aplicada.
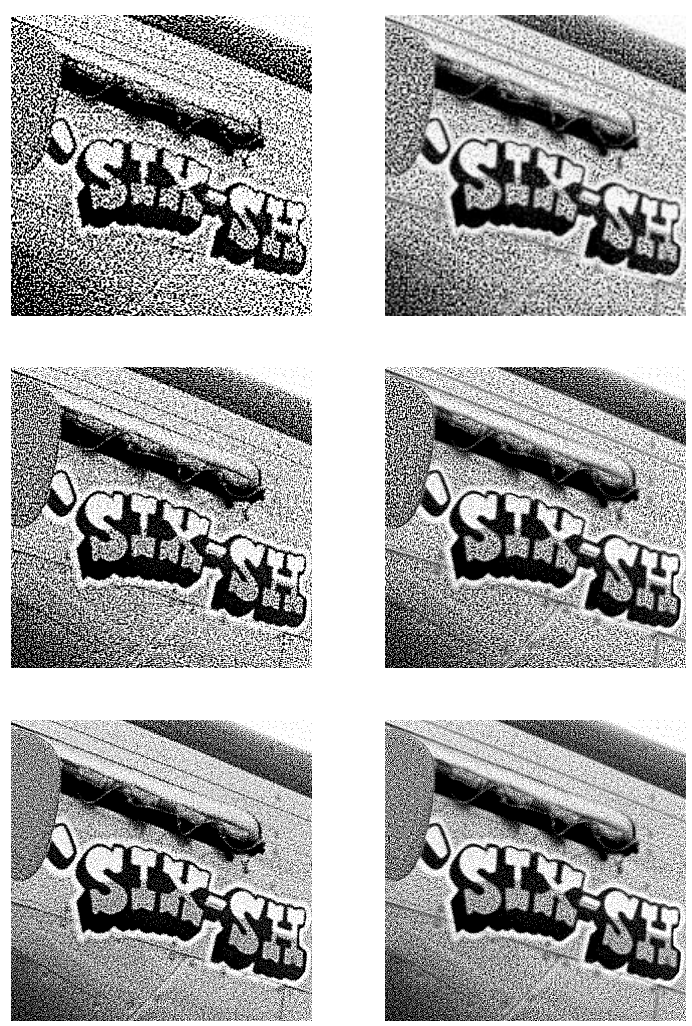

Figure 6: Comparison of adaptive (right) and non-adaptive (left) clustering. From top to bottom, cluster sizes of 30,15 , and 3 pixels

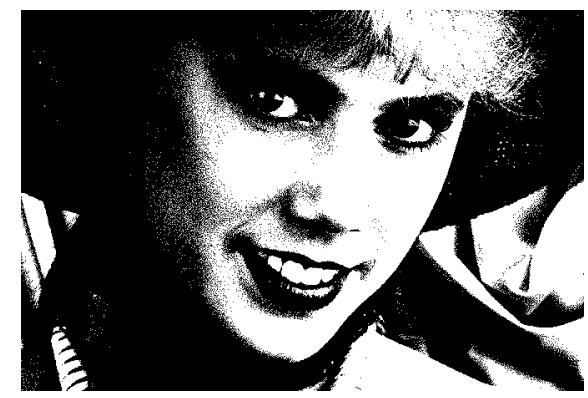

(a)

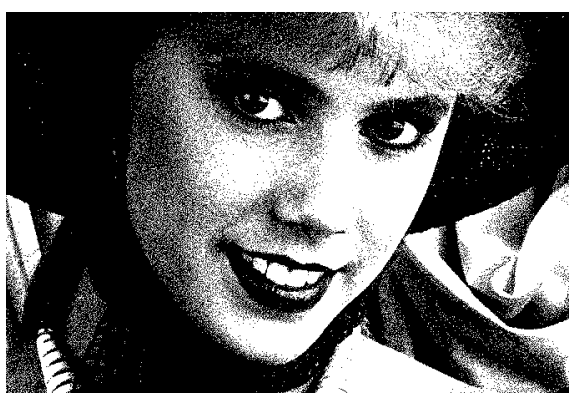

(b)

Figure 7: Images printed at a resolution of $1200 \mathrm{dpi}$ : (a) disperseddot dither, (b) adaptive dither with maximum cluster size of 7 pixels. 